\title{
Estrategias de tratamiento en la pancreatitis crónica: un enfoque del tratamiento quirúrgico
}

\author{
Jesús Morales-Maza*, Uriel Clemente-Gutiérrez y Óscar Santes \\ Departamento de Cirugía, Instituto Nacional de Ciencias Médicas y Nutrición Salvador Zubirán, Ciudad de México, México
}

\begin{abstract}
RESUMEN
La pancreatitis crónica es un padecimiento inflamatorio irreversible del páncreas con una repercusión importante en los pacientes que la sufren. Por tal motivo se han descrito múltiples formas de tratamiento que van desde el manejo conservador hasta procedimientos endoscópicos y quirúrgicos, con diferentes resultados cada uno. En los últimos años ha habido un gran avance científico en la búsqueda de la mejor estrategia terapéutica de esta entidad. El objetivo de esta publicación es realizar una revisión y actualización de los diferentes tipos de tratamiento de la pancreatitis crónica poniendo énfasis en el tratamiento quirúrgico. Se realizó una revisión de la literatura con la finalidad de actualizar el tema. Se seleccionaron 108 artículos. Se incluyeron metaanálisis, revisiones, estudios prospectivos y retrospectivos. Se utilizó la base de datos de Medline y se incluyeron artículos en idioma inglés usando los términos chronic pancreatitis combinados con pain, treatment, endoscopy, ESWL, surgery. Se seleccionaron artículos completos de los últimos 30 años.
\end{abstract}

Palabras clave: Pancreatitis crónica. Procedimientos quirúrgicos. Complicaciones. Cirugía. Páncreas.

\begin{abstract}
Chronic pancreatitis is an irreversible inflammatory disease of the pancreas with important repercussion in patients suffering from it, for this reason, multiple treatments have been described, ranging from conservative management to endoscopic and surgical procedures, with many different results. In recent years there has been a great scientific advance in the search for the best therapeutic strategy. The aim of this publication is to perform a review and update of the different types of treatment of chronic pancreatitis with an emphasis on surgical treatment. A review of literature was done in order to update the topic. We selected 108 articles. We included meta-analyses, reviews, prospective and retrospective studies. We used the MEDLINE database, we included articles in English using the terms "chronic pancreatitis" combined with "pain", "treatment", "endoscopy", "ESWL", "surgery". Full articles from the last 30 years were selected. (Hosp Med Clin Manag. 2018;11:36-46)

Corresponding author: Jesús Morales-Maza, medcardiouv@ hotmail.com
\end{abstract}

Key words: Pancreatitis. Chronic. Surgical procedures. Complications. Surgery. Pancreas.

\section{Correspondence:}

*Jesús Morales-Maza

Departamento de Cirugía

Instituto Nacional de Ciencias Médicas y Nutrición Salvador Zubirán

Vasco de Quiroga, 15

Col. Belisario Domínguez Sección XVI, Del. Tlalpan

C.P. 14080, Ciudad de México, México

E-mail: medcardiouv@ hotmail.com

Received for publication: 24-08-2017

Accepted for publication: 18-03-2018

DOI: $10.24875 / \mathrm{HMCM} .18000090$ 


\section{INTRODUCCIÓN}

La pancreatitis crónica es un padecimiento inflamatorio irreversible del páncreas que progresivamente se destruye y se acompaña de fibrosis. Se presenta en individuos con predisposición genética expuestos a múltiples factores de riesgo ambientales que desarrollan una respuesta patológica a una lesión parenquimatosa 0 al estrés ${ }^{1-3 .}$

Se caracteriza por tres procesos fisiopatogénicos:

- Hipertensión del conducto pancreático.

- Inflamación del páncreas.

- Infiltración de células de inmunidad con inflamación y fibrosis 4,5 .

Como consecuencia de dicha inflamación crónica, se presenta una destrucción irreversible del parénquima pancreático caracterizada clínicamente por dolor epigástrico recurrente en el $90 \%$ de los casos e insuficiencia exocrina (alteración de la digestión con diarrea) y endocrina (diabetes) con un aumento del riesgo de padecer cáncer de páncreas a largo plazo ${ }^{2,6,7}$. Además la calidad de vida de los pacientes resulta profundamente afectada ${ }^{8,9}$.

La incidencia de la pancreatitis crónica no es homogénea globalmente por la dificultad que representa la estandarización del diagnóstico, pero en general se ha estimado que es de 2 a 200 casos por cada 100,000 habitantes al año. Algunos países presentan una alta incidencia, como la India (114-200 casos por cada 100,000 habitantes), y otros países han presentado un aumento gradual de su incidencia y prevalencia ${ }^{10-14}$. Otra característica no homogénea es la diferente presentación clínica en los distintos grupos raciales ${ }^{15}$.

Todo esto da como resultado una alta carga de salud pública, con 327,000 hospitalizaciones, 200,000 visitas a Urgencias, 532,000 consultas médicas y un costo anual de 2.5 billones de dólares en EE.UU. Además se ha visto que estos pacientes presentan una alta tasa de readmisión hospitalaria ${ }^{16,17}$.

\section{ETIOLOGÍA DE LA PANCREATITIS CRÓNICA}

Actualmente se acepta como fisiopatogenia el antecedente de múltiples episodios de pancreatitis aguda que conllevan a largo plazo una pancreatitis crónica, y algunos autores han propuesto que el proceso crónico surge de un episodio de pancreatitis aguda inicial ${ }^{18,19}$.

Existen múltiples factores relacionados con la pancreatitis crónica, dentro de los cuales destacan el consumo de alcohol (70\% de los casos), los factores hereditarios (los factores genéticos más importantes son las mutaciones en el tripsinógeno catiónico PRSS1, inhibidor de serina proteasa tipo Kazal 1 y carboxipeptidasa $A 1$, entre otros), el hiperparatiroidismo, la hipertrigliceridemia, el traumatismo y las alteraciones congénitas anatómicas (páncreas divisum $)^{1,20-23}$

Se han realizado múltiples clasificaciones de la pancreatitis crónica, pero una manera simple de clasificarla de acuerdo a su etiología es dividiéndola de la siguiente manera ${ }^{24-26}$ :

- Pancreatitis alcohólica.

- Pancreatitis no alcohólica: idiopática, hereditaria, familiar, etc.

\section{MECANISMOS DEL DOLOR EN LA PANCREATITIS CRÓNICA}

El dolor abdominal es la manifestación más común de la pancreatitis crónica ${ }^{10}$. Se han descrito múltiples mecanismos de este síntoma ${ }^{27,28}$ :

- Alteración de la conducción nerviosa.

- Alteración de la nocicepción periférica.

- Neuropatía pancreática.

- Mecanismos de dolor central.

- Alteración de la presión del conducto pancréatico.

\section{PANORAMA GENERAL DEL TRATAMIENTO DE LA PANCREATITIS CRÓNICA}

Se han descrito múltiples tratamientos, desde el manejo conservador hasta procedimientos endoscópicos avanzados y procedimientos quirúrgicos, cada uno de 
Tabla 1. Diferentes factores a tratar en la pancreatitis crónica (manejo conservador)

Tratamiento de la causa

Tratamiento del dolor crónico

Tratamiento de la insuficiencia pancreática exocrina

Tratamiento de la insuficiencia pancreática endocrina

Terapia adyuvante

\section{Alcoholismo, tabaquismo ${ }^{31}$}

Analgesia basada en la escala del manejo del dolor de la OMS

Manejo con enzimas pancreáticas ${ }^{32}$

Dieta, antidiabéticos e insulina

Se han estudiado antioxidantes en el tratamiento de la pancreatitis crónica, pero algunos autores no apoyan su uso en dicha entidad por falta de suficiente evidencia para su utilización rutinaria ${ }^{33,34}$

OMS: Organización Mundial de la Salud.

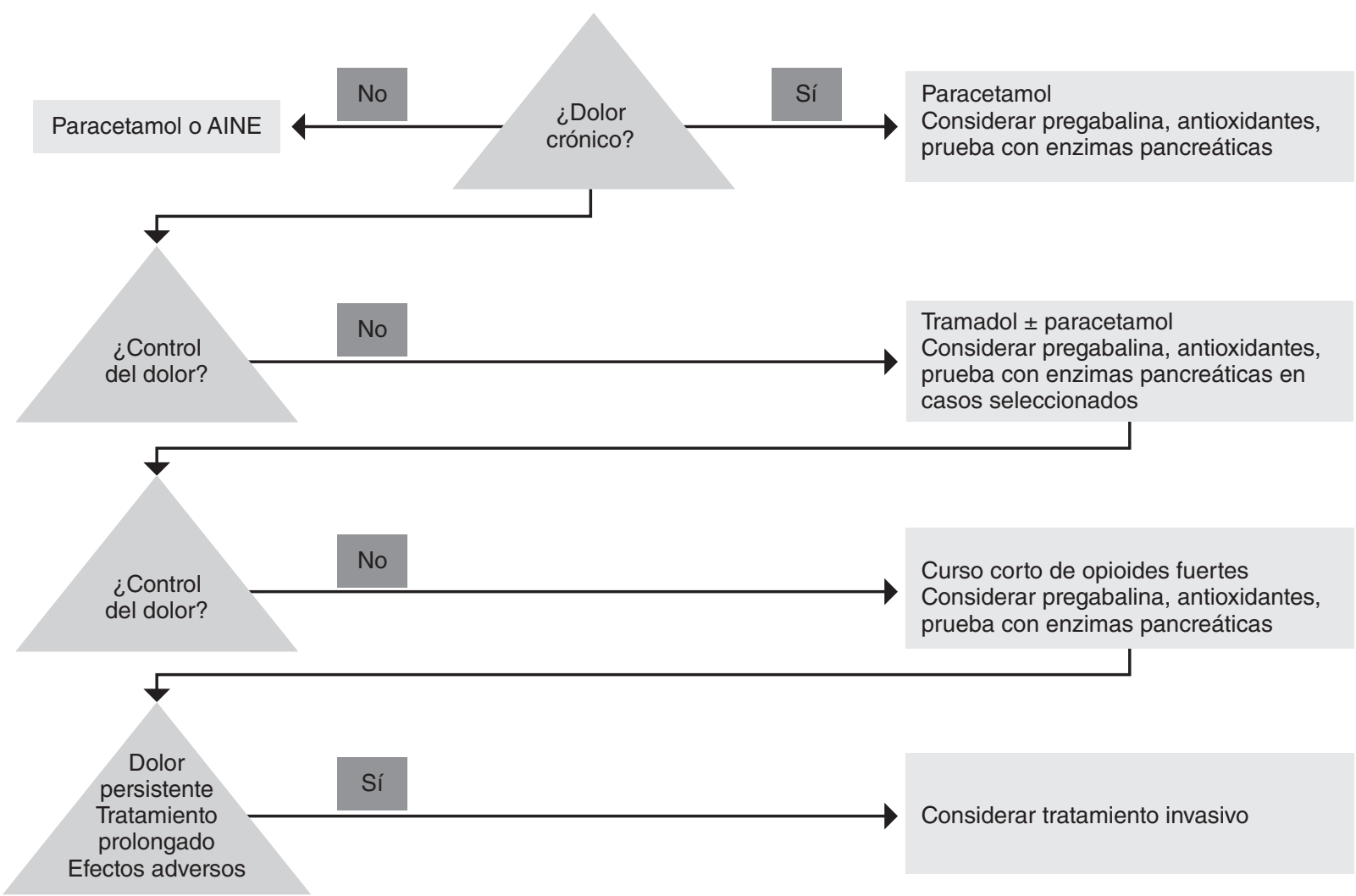

Figura 1. Propuesta de manejo del dolor de manera escalonada en pacientes con pancreatitis crónica (adaptado De-Madaria, et al. ${ }^{35}$ ). AINE: antiinflamatorios no esteroideos.

los cuales tiene cierto índice de éxito. Por tal motivo se sigue investigando la mejor estrategia de tratamiento de esta entidad. A continuación se describen los diferentes tratamientos de la pancreatitis crónica, haciendo énfasis en el tratamiento quirúrgico.

\section{Tratamiento conservador}

El tratamiento conservador sólo logra respuesta en el $31 \%$ de los $\operatorname{casos}^{29,30}$ y su principal objetivo es atenuar múltiples factores etiológicos para lograrlo (Tabla 1).

Se ha propuesto manejar el dolor de manera escalonada $(\text { Fig. } 1)^{35}$.
Se debe tener presente que el manejo conservador es frecuentemente insatisfactorio, con recurrencia de los síntomas a corto plazo, y muchos pacientes llegan a necesitar un tratamiento endoscópico o quirúrgico ${ }^{36}$.

\section{Tratamiento endoscópico}

Desde hace varias décadas la endoscopia se ha vuelto un tratamiento muy frecuentemente empleado en la pancreatitis crónica ${ }^{29}$. Sus objetivos son mejorar la calidad de vida y disminuir el dolor crónico ${ }^{7,37,38}$.

- Las principales indicaciones para realizar un tratamiento endoscópico de la pancreatitis crónica son 
las estenosis proximales sin masa inflamatoria y las pancreatitis de «conducto pequeño» (<3 mm de dilatación) $)^{39}$.

Se sabe que algunos tipos de pancreatitis crónica no se benefician del tratamiento endoscópico y se ha sugerido que deberían ir a cirugía de inicio, como las obstrucciones distales (conducto pancreático principal en la cola del páncreas), la presencia de múltiples calcificaciones pancreáticas o la presencia de masa inflamatoria, principalmente en la cabeza del páncreas, en la cual se vuelva difícil descartar la malignidad ${ }^{40}$

\section{EL ROL DE LA ENDOSCOPIA EN EL TRATAMIENTO DE LA PANCREATITIS CRÓNICA}

Existen varios tipos de tratamiento endoscópico y cada uno de ellos tiene una o varias indicaciones puntuales.

La descompresión endoscópica se emplea en el tratamiento de estenosis mediante el uso de stents y la extracción de litos en pacientes con el conducto pancreático dilatado; logra el alivio del dolor a corto plazo en el $74-94 \%$ de casos y a largo plazo, en el $52-83 \%{ }^{41}$. La litotripsia extracorpórea se emplea en casos de litos grandes mayores de $5 \mathrm{~mm}$ o que presentan dificultad para ser extraídos mediante una colangiopancreatografía retrógrada endoscópica; tiene éxito en el control del dolor en el $45 \%$ de casos aproximadamente ${ }^{42}$. Por último, la neurólisis del plexo celíaco guiada por ultrasonografía endoscópica se emplea cuando el conducto principal no presenta dilatación (se piensa que en estos casos la etiología del dolor es una inflamación peripancreática con afectación neural sin asociación con la dilatación del conducto pancreático principal) y logra el alivio del dolor a corto plazo en el 50-70\% de los casos y a largo plazo, sólo en el $10 \%{ }^{29}$.

Hay múltiples estudios que han valorado la utilidad del manejo de la pancreatitis crónica mediante endoscopia. En un estudio retrospectivo publicado en 2005 donde se incluyeron 343 pacientes con conducto principal dilatado el dolor desapareció en todos los pacientes en las primeras semanas, pero a los seis años sólo seis pacientes se encontraban sin dolor ${ }^{43}$. Otro estudio valoró la utilidad de la colocación de stents con un seguimiento a cinco años, y se observó que en 8 de 14 pacientes el dolor mejoró, pero 6 pacientes continuaron
Tabla 2. Indicaciones de tratamiento quirúrgico

- Falla al manejo conservador o endoscópico

- Dolor intratable 52

- Complicaciones locales sintomáticas como obstrucción de órganos adyacentes intratables con endoscopia o pseudoquistes (presente en el $20-40 \%$ de casos aproximadamente) $)^{53-55}$

- Sospecha de malignidad 56

con dolor y 2 requirieron cirugía para el tratamiento de la pancreatitis crónica ${ }^{44}$.

Hay algunos factores de buen pronóstico de respuesta al tratamiento endoscópico como los litos menores a 10 mm con ubicación en la cabeza y cuerpo del páncreas, cuando se trata de menos de tres litos y sin evidencia de múltiples estenosis ${ }^{45}$.

A pesar de contar con múltiples estrategias de tratamiento endoscópico para tratar la pancreatitis crónica y de que actualmente ya se ha demostrado que la cirugía en el tratamiento de la pancreatitis crónica es más efectiva a largo plazo ${ }^{46,47}$, el tratamiento endoscópico sigue teniendo una amplia utilidad en ciertos pacientes, y un gran porcentaje de éstos resolverán sus síntomas con la endoscopia sin llegar a requerir ningún tratamiento quirúrgico ${ }^{48}$.

\section{TRATAMIENTO QUIRÚRGICO DE LA PANCREATITIS CRÓNICA}

\section{El tratamiento quirúrgico de la pancreatitis crónica tiene} varios objetivos:

- Controlar el dolor.

- Mejorar la calidad de vida.

- Evitar la progresión de la enfermedad.

- Tratar las complicaciones ${ }^{49}$.

Cabe resaltar que a largo plazo el $40-75 \%$ de los pacientes con pancreatitis crónica necesitarán un tratamiento quirúrgico ${ }^{50,51}$.

Hay varias indicaciones para realizar un tratamiento quirúrgico en la pancreatitis crónica (Tabla 2).

Tradicionalmente los procedimientos quirúrgicos se han reservado para los pacientes que fracasan al tratamiento 
inicial endoscópico, y no se han considerado el tratamiento al inicio del diagnóstico ${ }^{57,58}$; sin embargo, algunos autores recientemente han asociado la cirugía temprana con un mejor pronóstico al presentar menos riesgo de insuficiencia pancreática a largo plazo y una menor cantidad de intervenciones. Por otro lado, se sabe que los pacientes con pancreatitis crónica presentan un leve riesgo de desarrollar un adenocarcinoma pancreático, cuya incidencia, según se ha visto, disminuye con la cirugía, pero aún falta evidencia para definir esta nueva estrategia ${ }^{59-63}$

\section{ANTECEDENTES HISTÓRICOS}

La historia del tratamiento quirúrgico de la pancreatitis crónica es muy amplia y hay personajes que no podemos dejar de mencionar, ya que su aportación individual hizo que evolucionara el conocimiento en esta área y se mejorara el pronóstico. En 1898 Gould removió un cálculo del conducto pancreático de un paciente y realizó un segundo procedimiento quirúrgico 17 días después para remover otro cálculo duodenal, pero el paciente murió 12 días después de la cirugía por «agotamiento». En 1908 Mayo y Robson describieron un abordaje transduodenal a través de la papila de Váter para extraer litos. En 1939 Haggard y Kirtley reportaron los casos de 65 pacientes a los que les retiraron litos intrapancreáticos y que presentaron una mortalidad del $18 \%$, pero no dieron un seguimiento a largo plazo ${ }^{64}$. En 1956 Doubilet realizó una esfinteroplastia en un intento de prevenir una pancreatitis crónica, pero esta estrategia no era exitosa en todos los casos, ya que, como se ha mencionado antes, la etiología de la pancreatitis crónica no siempre conlleva la presencia de litos. En 1911 el ginecólogo Goethe Link realizó la movilización del páncreas y drenaje pancreático de litos ${ }^{65}$.

El antecedente de drenaje del conducto pancreático para descomprimirlo proviene de la combinación del conocimiento aportado por César Roux y Dejardins; el primero, en 1897, describió la técnica para comunicar el yeyuno con el estómago durante la realización de gastrectomías y el segundo propuso en 1907 el uso de la derivación del conducto pancreático en perros. Así fue como Cattell en 1947 realizó una pancreatoyeyunoanastomosis laterolateral empleando la ahora conocida Y de Roux en el tratamiento paliativo del cáncer de páncreas ${ }^{66}$.

\section{DIFERENTES MÉTODOS DE TRATAMIENTO}

Se han descrito múltiples métodos quirúrgicos y progresivamente se ha logrado disminuir la morbilidad y mortalidad con una mejoría de los pronósticos a corto y largo plazo ${ }^{67}$.

De manera didáctica podemos clasificar los procedimientos de la siguiente manera:

- Procedimientos descompresivos.

- Procedimientos de resección.

- Procedimientos combinados.

- Procedimientos neuroablativos.

\section{Procedimientos descompresivos}

Este tipo de procedimiento se realiza cuando hay evidencia de conducto principal pancreático dilatado igual o mayor a 6-7 mm y sin evidencia de inflamación en la cabeza del páncreas (pseudotumor) y, por lo tanto, la hipertensión pancreática ductal puede ser la causa del dolor 68

Los tres procedimientos descompresivos descritos son el de Zollinger-DuVal, el de Puestow-Guillesby y el de Partington-Rochelle.

\section{Procedimiento de DuVal}

El procedimiento de DuVal se describió en 1954 como una resección de la cola pancreática y una anastomosis entre el páncreas y el yeyuno de manera terminoterminal. Este procedimiento sólo es aplicable a la obstrucción del conducto pancreático principal a nivel de la cabeza del páncreas con dilatación del resto del conducto, pero rara vez se realiza en nuestros días porque la anastomosis frecuentemente se obstruye y el dolor tiende a recurrir ${ }^{4}$.

\section{Procedimiento de Puestow-Guillesby}

El fundamento del procedimiento de Puestow original ha sido fundamental en el tratamiento de la pancreatitis crónica hasta nuestros días ${ }^{69}$. Fue descrito en 1958 por Puestow y Gillesby. En esta modificación del procedimiento de 
DuVal se realiza la resección de la cola pancreática, además de una esplenectomía, y se abre el conducto principal pancreático de manera longitudinal realizando una anastomosis entre el conducto pancreático y el yeyuno (terminoterminal) invaginando el páncreas dentro del yeyuno para evitar la fuga de la anastomosis. Este procedimiento estaba indicado principalmente en pacientes con múltiples estenosis a lo largo de todo el conducto pancreático principal y litos intraductales. No todos los pacientes se benefician de este procedimiento ya que no todos presentan una dilatación del conducto principal y, a pesar de tener muy buenos resultados a corto plazo (60-80\%), a los cinco años aproximadamente el $30 \%$ de pacientes presentan una recurrencia del dolor ${ }^{70}$

Desde hace algunos años se ha empezado a describir dicho procedimiento de manera laparoscópica y con cirugía robótica, pero todavía falta definir el verdadero costo-beneficio de estas nuevas estrategias $4,71-74$.

\section{Procedimiento de Partington-Rochelle (actualmente llamado procedimiento de Puestow)}

En 1960, dos años después de que se describiera la técnica de Puestow y Gillesby, Partington y Rochelle describieron una modificación de dicha técnica. Realizaron una apertura del conducto pancreático principal y una anastomosis entre el yeyuno y dicho conducto pero de manera laterolateral. Con esto lograron descomprimir toda la longitud del conducto principal de manera exitosa preservando el bazo y la cola del páncreas. Este procedimiento marcó el inicio de la «preservación de órgano» en la cirugía de la pancreatitis crónica. Aunque fue descrito por Partington y Rochelle, tradicionalmente se ha denominado procedimiento de Puestow y hoy día es el procedimiento descompresivo que más frecuentemente se realiza; la mayoría de los estudios que comparan los procedimientos quirúrgicos con el tratamiento endoscópico se basan en esta cirugía ${ }^{48}$. Es una técnica segura con una morbilidad y mortalidad muy bajas; además preserva la función pancreática endocrina y exocrina. A largo plazo logra controlar el dolor en el $80 \%$ de los casos $^{75}$.

\section{Procedimientos de resección}

Resecar de manera total o completa el parénquima pancreático es una forma atractiva de solucionar el problema del dolor en la pancreatitis crónica. Se han descrito múltiples procedimientos de resección, dentro de los cuales destacan el procedimiento de Whipple, descrito en 1935 para el tratamiento del cáncer pancreático, la pancreatoduodenectomía preservadora de píloro (Watson, 1944), la pancreatectomía distal, la pancreatectomía del segmento medio, el procedimiento de Beger y una modificación de este último procedimiento realizada por Imaizumi (Hamburg). A continuación se detallan los procedimientos mencionados.

\section{Procedimiento de Whipple y pancreatoduodenectomía preservadora del píloro}

El procedimiento de Whipple (y la pancreatoduodenectomía preservadora de píloro) se indica principalmente cuando existe predominio de inflamación en la cabeza del páncreas en pacientes sin evidencia de dilatación ductal («enfermedad de pequeño conducto») o en pacientes en quienes se sospecha que podría existir un adenocarcinoma de la cabeza del páncreas. La técnica quirúrgica del Whipple clásica incluye la realización de la resección de la cabeza del páncreas, el duodeno y una porción del estómago distal. La técnica de la pancreatoduodenectomía preservadora del píloro fue descrita con la intención de realizar un procedimiento más «fisiológico» sin resecar el píloro y mejorar el vaciamiento gástrico. En esencia es el mismo procedimiento; la diferencia radica en que se realiza la sección del duodeno a $2 \mathrm{~cm}$ distal al píloro preservándolo.

Ambos procedimientos han presentado resultados favorables a corto y largo plazo, de aproximadamente el $80-100 \%$, con mortalidad menor del $2 \%$, pero con una morbilidad posoperatoria que va del 12 al 33\% (fístula pancreática, retraso del vaciamiento gástrico y colangitis), con desarrollo de diabetes en aproximadamente el $20 \%$ de los pacientes ${ }^{76-78}$.

\section{Pancreatectomía total}

La pancreatectomía total fue descrita inicialmente en 1944 para el tratamiento de un insulinoma, pero su uso se abandonó debido a la alta frecuencia de complicaciones. A pesar de ello, hay reportes de tratamiento de la pancreatitis crónica empleando este procedimiento que han mostrado buenos resultados en el control del dolor y que presenta su mayor utilidad en pacientes con afectación pancreática difusa, como procedimiento de 
"salvamento» cuando fallan otros procedimientos quirúrgicos, cuando se presentan complicaciones como fístulas pancreáticas o fuga de anastomosis, e incluso algunos autores han sugerido su utilidad en la pancreatitis hereditaria ${ }^{79}$. Sin embargo, por razones obvias se espera que los pacientes presenten una insuficiencia pancreática y por eso esta intervención está contraindicada como primera línea de tratamiento80-82.

\section{Pancreatectomía distal}

La pancreatectomía distal se describió en 1931 y se ha indicado en pacientes con predominio de inflamación localizada a nivel del cuerpo y la cola del páncreas o complicaciones locales de la cola pancreática como pseudoquistes. Los resultados van del 55 al $81 \%$ en el control de dolor. A pesar de esto, una de sus complicaciones temidas es la fístula pancreática (en más del 10\% de los casos). Además la resección pancreática distal frecuentemente se asocia a una insuficiencia pancreática endocrina y exocrina ${ }^{83}$

\section{Pancreatectomía del segmento medio}

La pancreatectomía del segmento medio se describió inicialmente en el tratamiento de tumores localizados en el cuello del páncreas, pero también se ha empleado en el tratamiento de la pancreatitis crónica. Esta técnica no ha ganado popularidad como el resto de los procedimientos descritos ${ }^{84}$.

\section{Procedimientos combinados}

Esta categoría se refiere a la combinación de resección del parénquima pancreático más drenaje o descompresión del conducto pancreático principal. Destacan en este grupo el procedimiento de Beger, el de Frey, una modificación del procedimiento de Frey llamada Hamburg, el procedimiento de Berne y el de Imaizumi.

\section{Procedimiento de Beger (resección de la cabeza del páncreas preservadora del duodeno)}

Con el fundamento de que la pancreatitis crónica presenta predominio de inflamación en la cabeza del páncreas («marcapasos de la pancreatitis crónica») surgieron nuevas técnicas que trataban de enfocar la resección a dicha zona con el fin de resecar sólo la porción «enferma» del páncreas preservando zonas que, en teoría, si son resecadas no favorecen la mejoría del dolor pero sí contribuyen al desarrollo de una insuficiencia pancreática, incluyéndose también la preservación del duodeno. En comparación con procedimientos más radicales (como el de Whipple), el paso gastroduodenal y la continuidad del colédoco son preservados $^{85}$.

La primera cirugía de resección preservadora de duodeno fue descrita en 1980 por Beger y mostró buenos resultados a largo plazo. En múltiples estudios se ha comparado dicho procedimiento con el de Frey, el de Berne y la pancreatoduodenectomía preservadora de píloro, y se han evidenciado resultados muy parecidos a largo plazo en el control del dolor entre todos los procedimientos ${ }^{86-90}$

\section{Procedimiento de Frey}

El procedimiento de Frey, una modificación del de Beger, combina la resección local de la cabeza del páncreas con la pancreatoyeyunostomía longitudinal y desde su descripción en 1987 ha sido estudiado y comparado con los diferentes procedimientos quirúrgicos mostrando resultados similares ${ }^{91}$ con buen control del dolor (62-90\%) y una mortalidad menor al $2 \%$. Algunas complicaciones como la insuficiencia exocrina y endocrina se presentan en el 70 y el $8-34 \%$ de los casos, respectivamente ${ }^{92,93}$. En algunos centros ya se empieza a describir su realización laparoscópica ${ }^{94,95}$. En este procedimiento sólo se excava la cabeza del páncreas y se realiza una pancreaticoyeyunoanastomosis directa sobre dicha excavación sin realizar la exposición del conducto pancreático principal96.

\section{Modificación de Berne}

Posteriormente se describió un procedimiento de resección de la cabeza del páncreas con preservación del duodeno en pacientes con cirrosis hepática (procedimiento de Berne), y a partir de esta modificación se fundamentó otra modificación con excavación de la porción central de la cabeza del páncreas sin incluir el conducto pancreático en la pancreatoyeyunoanastomosis lateral ${ }^{97,98}$.

\section{Procedimiento de Hamburg}

El procedimiento de Hamburg, descrito en 1998, es una combinación del procedimiento de Frey con una 
resección local longitudinal de la cabeza del páncreas en forma de V. Sus autores sugieren emplearlo en pacientes con pancreatitis crónica de "conducto pequeño» con el beneficio de realizar una resección preservadora del órgano con el drenaje de los conductos primarios y secundarios y al mismo tiempo mantener órgano suficiente para preservar la función pancreática. Se han descrito buenos resultados a largo plazo en el control del dolor (75\%) y la mejoría de la calidad de vida de los pacientes. Además el $78 \%$ de los pacientes preservan la función pancreática exocrina, pero el $43 \%$ desarrollan diabetes ${ }^{99-101}$.

\section{Procedimiento de Imaizumi}

En el año 2009 Hatori describió una modificación del procedimiento de Beger con utilidad en pacientes con estenosis del colédoco intrapancreático ${ }^{102}$. Prácticamente es una combinación del procedimiento de Beger con una resección pancreática preservadora del duodeno. Se ha descrito control del dolor en el $90 \%$ de los casos y menor incidencia de insuficiencia pancreática que con la pancreatoduodenectomía preservadora de píloro ${ }^{4}$.

\section{Procedimientos neuroablativos}

Anatómicamente existe un amplio plexo que inerva el páncreas; por eso se han propuesto múltiples alteraciones nerviosas como causa del dolor crónico ${ }^{27}$. A partir de este conocimiento se ha intentado mitigar el dolor alterando estas vías de conducción. Principalmente se han considerado los procedimientos neuroablativos en pacientes sin dilatación del conducto pancreático y sin litiasis intraductal en los cuales se podría pensar que la causa del dolor no es la dilatación del conducto principal, sino una alteración nerviosa aferente. Sin embargo, se ha tenido poco éxito a largo plazo, y por eso no se ha generalizado su utilización de manera rutinaria.

Como antecedente, en 1947 Bronson Ray efectuó una simpatectomía bilateral y se realizó de manera intraoperatoria el estímulo eléctrico sin evidencia de dolor, pero no se dio seguimiento del resultado a largo plazo ${ }^{103}$. En 1979 se reportó un estudio con un seguimiento de ocho años en promedio y mejoría del dolor en 3 de 22 pacientes con esplanicectomía celíaca ${ }^{104}$. En 1984 Warren, et al. realizaron una denervación esplenopancreática con resección de la cabeza pancreática, pero no reportaron los resultados a largo plazo ${ }^{105}$.
Otras medidas neuroablativas para tratar la pancreatitis crónica son el bloqueo del plexo celíaco y la esplanicectomía toracoscópica bilateral, con buenos resultados a corto plazo (50-60\%). No obstante, con el paso de las semanas o meses el dolor regresa (recurrencia del dolor en el $50 \%$ de los casos a los 15 meses de seguimiento). Por tal motivo las denervaciones quirúrgicas no se consideran un tratamiento quirúrgico de primera línea65,106.

\section{OTROS PROCEDIMIENTOS}

\section{Pancreatectomía total con autotrasplante de islotes pancreáticos}

En 1980 se demostró la utilidad de este procedimiento en el tratamiento de la pancreatitis crónica, principalmente con el objetivo de evitar la dependencia de la insulina a largo plazo. La técnica incluye la preservación de la glándula con cultivo de células pancreáticas y la infusión de éstas dentro de la vena porta. Se requieren de 300,000 a 400,000 islotes para lograr injertar con éxito. La cantidad de islotes que se obtienen generalmente depende del grado de enfermedad presente. Desafortunadamente el $25-30 \%$ de los pacientes con pancreatitis crónica son diabéticos y, por lo tanto, no se consideran candidatos para la realización de este procedimiento65.

Una de las desventajas de este procedimiento es su costo y además a largo plazo un gran porcentaje de pacientes utilizarán insulina a pesar del injerto. Cabe resaltar que esta estrategia terapéutica presenta un buen resultado en el control del dolor a largo plazo ${ }^{107}$.

\section{Selección de la cirugía}

Son múltiples los factores a tomar en cuenta para seleccionar la cirugía a realizar en cada paciente, como, por ejemplo, la evidencia de masa pancreática, la obstrucción del conducto principal y la evidencia de complicaciones de la pancreatitis crónica como la presencia de pseudoquiste pancreático, pero hasta ahora no se ha estandarizado el tratamiento de los pacientes ni el tipo de cirugía a realizar, y la tendencia actual es individualizar cada caso ${ }^{108}$.

\section{CONCLUSIONES}

El manejo de la pancreatitis crónica debe ser multidisciplinario, ya que existen múltiples tratamientos con 
diferentes resultados, y esto mejora el pronóstico del paciente.

El tratamiento de la pancreatitis crónica se debe ajustar de manera individual a las características cada paciente. En aquéllos en los que se decida realizar la cirugía la tendencia es realizar procedimientos «preservadores de órgano».

A la fecha actual algunos estudios sugieren un mejor resultado de la cirugía temprana que de la tardía, pero aún falta por definir si de manera rutinaria todo paciente debe ir de inicio a cirugía. Los procedimientos endoscópicos han mostrado también buenos resultados y tienen el beneficio de ser más conservadores, así que por ahora la gran mayoría de los pacientes se benefician del tratamiento endoscópico de inicio para valorar la respuesta y, en caso de fracaso terapéutico, realización de cirugía.

\section{CONFLICTO DE INTERESES}

Los autores declaran no tener conflicto de intereses en esta publicación.

\section{BIBLIOGRAFÍA}

1. Conwell DL, Lee LS, Yadav D, Longnecker DS, Miller FH, Mortele KJ, et al. American Pancreatic Association Practice Guidelines in Chronic Pancreatitis: evidence-based report on diagnostic guidelines. Pancreas. 2014;43(8):1143-62.

2. Drewes AM. Understanding and treatment of chronic pancreatitis. World $\mathrm{J}$ Gastroenterol. 2013;19(42):7219-21.

3. Whitcomb DC, Frulloni L, Garg P, Greer JB, Schneider A, Yadav D, et al. Chronic pancreatitis: an international draft consensus proposal for a new mechanistic definition. Pancreatology. 2016;16(2):218-24.

4. Ni Q, Yun L, Roy M, Shang D. Advances in surgical treatment of chronic pancreatitis. World J Surg Oncol. 2015;13:34

5. Babu V. Image in clinical medicine. Chronic Calcific Pancreatitis. N Engl J Med. 2007;356(23):e24.

6. D'Haese JG, Ceyhan GO, Demir IE, Tieftrunk E, Friess H. Treatment options in painful chronic pancreatitis: a systematic review. HPB (Oxford). 2014;16(6):512-21.

7. Braganza JM, Lee SH, McCloy RF, McMahon MJ. Chronic pancreatitis. Lancet. 2011;377(9772):1184-97.

8. Gardner TB, Kennedy AT, Gelrud A, Banks PA, Vege SS, Gordon SR, et al. Chronic pancreatitis and its effect on employment and health care experience: results of a prospective American multicenter study. Pancreas. 2010;39(4):498-501.

9. Wassef W, DeWitt J, McGreevy K, Wilcox M, Whitcomb D, Yadav D, et al. Pancreatitis quiality of life instrument: a psychometric evaluation. Am J Gastroenterol. 2016;111(8):1177-86.

10. Lévy P, Domínguez-Muñoz E, Imrie C, Löhr M, Maisonneuve P. Epidemiology of chronic pancreatitis: burden of the disease and consequences. United European Gastroenterol J. 2014;2(5):345-54.

11. Yadav D, Timmons L, Benson JT, Dierkhising RA, Chari ST. Incidence, prevalence, and survival of chronic pancreatitis: a population-based study. Am J Gastroenterol. 2011;106(12):2192-9.

12. Machicado JD, Yadav D. Epidemiology of recurrent acute and chronic pancreatitis: similarities and differences. Dig Dis Sci. 2017;62(7):1683-91.

13. Lévy P, Barthet M, Mollard BR, Amouretti M, Marion-Audibert AM, Dyard F. Estimation of the prevalence and incidence of chronic pancreatitis and its complications. Gastroenterol Clin Biol. 2006;30(6-7):838-44.
14. Hirota M, Shimosegawa T, Masamune A, Kikuta K, Kume K, Hamada S, et al. The sixth nationwide epidemiological survey of chronic pancreatitis in Japan. Pancreatology. 2012;12(2):79-84.

15. Wilcox CM, Sandhu BS, Singh V, Gelrud A, Abberbock JN, Sherman S, et al. Racial differences in the clinical profile, causes, and outcome of chronic pancreatitis. Am J Gastroenterol. 2016;111(10):1488-96.

16. Lowenfels $A B$, Sullivan T, Fiorianti J, Maisonneuve P. The epidemiology and impact of pancreatic diseases in the United States. Curr Gastroenterol Rep. 2005;7(2):90-5

17. Fisher AV, Sutton JM, Wilson GC, Hanseman DJ, Abbott DE, Smith MT, et al. High readmission rates after surgery for chronic pancreatitis. Surgery. 2014;156(4):787-94

18. Comfort HW, Gambill EE, Baggenstoss AH. Chronic relapsing pancreatitis: a study of 29 cases without associated disease of the biliary or gastrointestinal tract. Gastroenterology. 1946:6:239-85.

19. Apte MV, Wilson JS. Alcohol-induced pancreatic injury. Best Pract Res Clin Gastroenterol. 2003;17(4):593-612.

20. Löhr JM, Dominguez-Munoz E, Rosendahl J, Besselink M, Mayerle J, Lerch MM, et al. United European Gastroenterology evidence-based guidelines for the diagnosis and therapy of chronic pancreatitis (HaPanEU). United European Gastroenterol J. 2017:5(2):153-99.

21. Zator Z, Whitcomb DC. Insights into the genetic risk factors for the development of pancreatic disease. Therap Adv Gastroenterol. 2017;10(3):323-36.

22. Manohar M, Verma AK, Venkateshaiah SU, Sanders NL, Mishra A. Pathogenic mechanisms of pancreatitis. World J Gastrointest Pharmacol Ther. 2017;8(1):10-25.

23. Stram M, Liu S, Singhi AD. Chronic Pancreatitis.Surg Pathol Clin. 2016;9(4):643-59.

24. Shimosegawa T, Kataoka K, Kamisawa T, Miyakawa H, Ohara H, Ito T, et al. The revised Japanese clinical diagnostic criteria for chronic pancreatitis. J Gastroenterol. 2010;45(6):584-91.

25. Schneider A, Löhr JM Singer MV. The M-ANNHEIM classification of chronic pancreatitis: introduction of a unifying classification system based on a review of previous classifications of the disease. $J$ Gastroenterol. 2007;42(2):101-19.

26. Etemad B, Whitcomb DC. Chronic pancreatitis: diagnosis, classification, and new genetic developments. Gastroenterology. 2001;120(3):682-707.

27. Poulsen JL, Olesen SS, Malver LP, Frøkjær JB, Drewes AM. Pain and chronic pancreatitis: A complex interplay of multiple mechanisms. World J Gastroenterol 2013;19(42):7282-91.

28. Pasricha PJ. Unraveling the mystery of pain in chronic pancreatitis. Nat Rev Gastroenterol Hepatol. 2012;9(3):140-51.

29. Jabło囚ska B. Is endoscopic therapy the treatment of choice in all patients with chronic pancreatitis? World J Gastroenterol. 2013;19(1):12-6.

30. Clarke B, Slivka A, Tomizawa Y, Sanders M, Papachristou GI, Whitcomb DC, et al. Endoscopic therapy is effective for patients with chronic pancreatitis. Clin Gastroenterol Hepatol. 2012;10(7):795-802.

31. Maisonneuve P, Frulloni L, Müllhaupt B, Faitini K, Cavallini G, Lowenfels AB, et al. Impact of smoking on patients with idiopathic chronic pancreatitis. Pancreas. 2006:33(2):163-8.

32. Issa Y, Bruno MJ, Bakker OJ, Besselink MG, Schepers NJ, van Santvoort $\mathrm{HC}$, et al. Treatment options for chronic pancreatitis. Nat Rev Gastroenterol Hepatol. 2014;11(9):556-64.

33. Strobel 0, Büchler MW, Werner J. Surgical therapy of chronic pancreatitis: indications, techniques and results. Int J Surg. 2009;7(4):305-12.

34. Forsmark CE, Liddle RA. The challenging task of treating painful chronic pancreatitis. Gastroenterology. 2012;143(3):533-5

35. de-Madaria E, Abad-González A, Aparicio JR, Aparisi L, Boadas J, Boix E, et al. The Spanish Pancreatic Club's recommendations for the diagnosis and treatment of chronic pancreatitis: part 2 (treatment). Pancreatology. 2013;13(1):18-28

36. Bhardwaj P, Garg PK, Maulik SK, Saraya A, Tandon RK, Acharya SK. A randomized controlled trial of antioxidant supplementation for pain relief in patients with chronic pancreatitis. Gastroenterology. 2009;136(1):149-59.e2.

37. Li ZS, Wang W, Liao Z, Zou DW, Jin ZD, Chen J, et al. A long-term followup study on endoscopic management of children and adolescents with chronic pancreatitis. Am J Gastroenterol. 2010;105(8):1884-92.

38. Li ZS, Wang W, Liao Z, Zou DW, Jin ZD, Chen J, et al. Endoscopic managements in pain of chronic pancreatitis. Chin J Dig Endosc. 2008;25:295-8.

39. Dumonceau JM, Macias-Gomez C. Endoscopic management of complications of chronic pancreatitis. World J Gastroenterol. 2013;19(42):7308-15.

40. Kahl S, Zimmermann S, Genz I, Glasbrenner B, Pross M, Schulz HU, et al. Risk factors for failure of endoscopic stenting of biliary strictures in chronic pancreatitis: a prospective follow-up study. Am J Gastroenterol. 2003;98(11):2448-53.

41. Seicean A, Vultur S. Endoscopic therapy in chronic pancreatitis: current perspectives. Clin Exp Gastroenterol. 2014:8:1-11.

42. Brand B, Kahl M, Sidhu S, Nam VC, Sriram PV, Jaeckle S, et al. Prospective evaluation of morphology, function, and quality of life after extracorporeal shockwave lithotripsy and endoscopic treatment of chronic calcific pancreatitis. Am J Gastroenterol. 2000;95(12):3428-38. 
43. Gabbrielli A, Pandolfi M, Mutignani M, Spada C, Perri V, Petruzziello L, et al. Efficacy of main pancreatic-duct endoscopic drainage in patients with chronic pancreatitis, continuos pain, and dilated duct. Gastrointest Endosc. 2005;61(4):576-81.

44. Weber A, Schneider J, Neu B, Meining A, Born P, von Delius S, et al. Endoscopic stent therapy in patients with chronic pancreatitis: A 5-year follow-up study. World J Gastroenterol. 2013;19(5):715-20.

45. Tringali A, Boskoski I, Costamagna $G$. The role of endoscopy in the therapy of chronic pancreatitis. Best Pract Res Clin Gastroenterol. 2008;22(1):145-65.

46. Cahen DL, Gouma DJ, Nio Y, Rauws EA, Boermeester MA, Busch OR, et al. Endoscopic versus surgical drainage of the pancreatic duct in chronic pancreatitis. N Engl J Med. 2007;356(7):676-84.

47. Díte P, Ruzicka M, Zboril V, Novotný I. A prospective, randomized trial comparing endoscopic and surgical therapy for chronic pancreatitis. Endoscopy. 2003:35(7):553-8.

48. Cahen DL, Gouma DJ, Laramée P, Nio Y, Rauws EA, Boermeester MA, et al. Long-term outcomes of endoscopic vs surgical drainage of the pancreatic duct in patients with chronic pancreatitis. Gastroenterology. 2011; 141(5):1690-5.

49. Nealon WH, Matin S. Analysis of surgical success in preventing recurrent acute exacerbations in chronic pancreatitis. Ann Surg. 2001:233(6):793-800.

50. Friess H, Berberat PO, Wirtz M, Büchler MW. Surgical treatment and longterm follow-up in chronic pancreatitis. Eur $J$ Gastroenterol Hepatol. 2002;14(9):971-7.

51. Layer $P$, Yamamoto $H$, Kalthoff $L$, Clain JE, Bakken LJ, DiMagno EP, et al. The different courses of early- and late-onset idiopathic and alcoholic chronic pancreatitis. Gastroenterology. 1994;107(5):1481-7.

52. Schnelldorfer T, Lewin DN, Adams DB. Operative management of chronic pancreatitis: longterm results in 372 patients. J Am Coll Surg. 2007;204(5):1039-45; discussion 1045-7.

53. Uiterwaal MT, Overbosch EH, Bruno MJ, van der Hulst RW. Spontaneous drainage of a pancreatic pseudocyst after embolization of a bleeding pseudoaneurysm. Cardiovasc Intervent Radiol. 2009;32(1):192-4.

54. Mihaljevic AL, Kleeff J, Friess H, Büchler MW, Beger HG. Surgical approaches to chronic pancreatitis. Best Pract Res Clin Gastroenterol. 2008;22(1):167-81.

55. da Cunha JE, Machado M, Bacchella T, Penteado S, Mott CB, Jukemura J, et al. Surgical treatment of pancreatic ascites and pancreatic pleural effusions. Hepatogastroenterology. 1995;42(5):748-51.

56. Liao Z, Jin G, Cai D. Guidelines: diagnosis and therapy for chronic pancreatitis. J Interv Gastroenterol. 2013;3(4):133-6.

57. Bachmann K, Izbicki JR, Yekebas EF. Chronic pancreatitis: modern surgical management. Langenbecks Arch Surg. 2011;396(2):139-49.

58. Warshaw AL, Banks PA, Fernández-Del Castillo C. AGA technical review: treatment of pain in chronic pancreatitis. Gastroenterology. 1998; 115(3):765-76.

59. Yang CJ, Bliss LA, Schapira EF, Freedman SD, Ng SC, Windsor JA, et al. Systematic review of early surgery for chronic pancreatitis: impact on pain, pancreatic function, and re-intervention. J Gastrointest Surg. 2014:18(10):1863-9.

60. Lamme B, Boermeester MA, Straatsburg IH, van Buijtenen JM, Boerma D, Offerhaus GJ, et al. Early versus late surgical drainage for obstructive pancreatitis in an experimental model. Br J Surg. 2007;94(7):849-54.

61. Nealon WH, Thompson JC. Progressive loss of pancreatic function in chronic pancreatitis is delayed by main pancreatic duct decompression. A longitudinal prospective analysis of the modified puestow procedure. Ann Surg. 1993:217(5):458-66; discussion 466-8.

62. Ahmed Ali U, Issa Y, Bruno MJ, van Goor H, van Santvoort H, Busch OR, et al. Early surgery versus optimal current step-up practice for chronic pancreatitis (ESCAPE): design and rationale of a randomized trial. BMC Gastroenterol. 2013:13:49

63. Ueda J, Tanaka M, Ohtsuka T, Tokunaga S, Shimosegawa T. Surgery for chronic pancreatitis decreases the risk for pancreatic cancer: a multicenter retrospective analysis. Surgery. 2013:153(3):357-64.

64. Haggard WD, Kirtley JA. Pancreatic calculi: a review of sixty-five operative and one hundred thirty-nine non-operative cases. Ann Surg. 1939; 109(5):809-26.

65. Hartmann D, Friess H. Surgical Approaches to Chronic Pancreatitis. Gastroenterol Res Pract. 2015;2015:503109.

66. Andersen DK, Frey CF. The Evolution of the Surgical Treatment of Chronic Pancreatitis. Ann Surg. 2010:251(1):18-32.

67. D'Haese JG, Ceyhan GO, Demir IE, Tieftrunk E, Friess H. Treatment options in painful chronic pancreatitis: a systematic review. HPB (Oxford). 2014;16(6):512-21.

68. Gourgiotis S, Dimopoulos N, Germanos S, Vougas V, Alfaras P, Hadjiyannakis E. Surgical management of chronic pancreatitis. Hepatobiliary Pancreat Dis Int. 2007;6(2):121-33.

69. Adams DB. The Puestow procedure: how I do it. J Gastrointest Surg. 2013:17(6):1138-42

70. Mannell A, Adson MA, Mcllrath DC, Ilstrup DM. Surgical management of chronic pancreatitis: long-term results in 141 patients. $\mathrm{Br} J$ Surg. 1988;75(5):467-72.
71. Biteman BR, Harr JN, Brody F. Laparoscopic Puestow: lateral pancreaticojejunostomy. Surg Endosc. 2016;30(12):5624

72. Crisanto-Campos BA, Trejo-Ávila ME, Román Ramírez E, Lina-López LM, Moreno-Portillo M. Total laparoscopic pancreaticojejunal anastomosis (modified Puestow procedure): A case report. Rev Gastroenterol Mex. 2017;82(1):97-9.

73. Khaled YS, Ammori BJ. Laparoscopic lateral pancreaticojejunostomy and laparoscopic Berne modification of Beger procedure for the treatment of chronic pancreatitis: the first UK experience. Surg Laparosc Endosc Percutan Tech. 2014;24(5):e178-82.

74. Meehan JJ, Sawin R. Robotic lateral pancreaticojejunostomy (Puestow). J Pediatr Surg. 2011;46(6):e5-8

75. Greenlee HB, Prinz RA, Aranha GV. Long-term results of side-to-side pancreaticojejunostomy. World J Surg. 1990;14(1):70-6.

76. Jimenez RE, Fernandez-del Castillo C, Rattner DW, Chang Y, Warshaw AL. Outcome of pancreaticoduodenectomy with pylorus preservation or with antrectomy in the treatment of chronic pancreatitis. Ann Surg. 2000;231(3):293-300.

77. Lü WP, Shi Q, Zhang WZ, Cai SW, Jiang K, Dong JH. A meta-analysis of the long-term effects of chronic pancreatitis surgical treatments: duodenum-preserving pancreatic head resection versus pancreatoduodenectomy. Chin Med J (Engl). 2013;126(1):147-53

78. Friess H, Berberat PO, Wirtz M, Büchler MW. Surgical treatment and longterm follow-up in chronic pancreatitis. Eur $\mathrm{J}$ Gastroenterol Hepatol. 2002:14(9):971-7.

79. Strate T, Knoefel WT, Yekebas E, Izbicki JR. Chronic pancreatitis: etiology, pathogenesis, diagnosis, and treatment. Int J Colorectal Dis. 2003;18(2): 97-106.

80. Behrman SW, Mulloy M. Total pancreatectomy for the treatment of chronic pancreatitis: indications, outcomes, and recommendations. Am Surg. 2006;72(4):297-302

81. Klaiber U, Alldinger I, Probst P. Bruckner T, Contin P, Köninger J, et al. Duodenum-preserving pancreatic head resection: 10-year follow-up of a randomized controlled trial comparing the Beger procedure with the Berne modification. Surgery. 2016;160(1):127-35.

82. Vasile D, Ilco A, Popa D, Belega A, Pana S. The surgical treatment of chronic pancreatitis: a clinical series of 17 cases. Chirurgia (Bucur). 2013;108(6):794-9.

83. Hutchins RR, Hart RS, Pacifico M, Bradley NJ, Williamson RC. Long-term results of distal pancreatectomy for chronic pancreatitis in 90 patients. Ann Surg. 2002;236(5):612-8.

84. Bassi C. Middle segment pancreatectomy: a useful tool in the management of pancreatic neoplasms. J Gastrointest Surg. 2007:11(4):421-4.

85. Izbicki JR, Bloechle C, Knoefel WT, Wilker DK, Dornschneider G, Seifert H, et al. Complications of adjacent organs in chronic pancreatitis managed by duodenum-preserving resection of the head of the pancreas. $\mathrm{Br} \mathrm{J}$ Surg. 1994;81(9):1351-5.

86. Bachmann K, Tomkoetter L, Erbes J, Hofmann B, Reeh M, Perez D, et al. Beger and Frey Procedures for Treatment of Chronic Pancreatitis: Comparison of Outcomes at 16-Year Follow-Up. J Am Coll Surg. 2014:219(2):208-16.

87. King JC, Abeywardina S, Farrell JJ, Reber HA, Hines OJ. A modern review of the operative management of chronic pancreatitis. Am Surg. 2010:76(10):1071-4

88. Izbicki JR, Bloechle C, Knoefel WT, Kuechler T, Binmoeller KF, Broelsch CE. Duodenum-preserving resection of the head of the pancreas in chronic pancreatitis. A prospective, randomized trial. Ann Surg. 1995; 221(4):350-8.

89. Müller MW, Friess H, Martin DJ, Hinz U, Dahmen R, Büchler MW. Long-term follow-up of a randomized clinical trial comparing Beger with pyloruspreserving Whipple procedure for chronic pancreatitis. $\mathrm{Br} J$ Surg 2008:95(3):350-6.

90. Diener MK, Rahbari NN, Fischer L, Antes G, Büchler MW, Seiler CM. Duodenum-preserving pancreatic head resection versus pancreatoduodenectomy for surgical treatment of chronic pancreatitis: a systematic review and meta-analysis. Ann Surg. 2008;247(6):950-61.

91. Strate T, Bachmann K, Busch P, Mann O, Schneider C, Bruhn JP, et al. Resection vs drainage in treatment of chronic pancreatitis: long-term results of a randomized trial. Gastroenterology. 2008;134(5):1406-11.

92. Frey CF, Mayer KL. Comparison of local resection of the head of the pancreas combined with longitudinal pancreaticojejunostomy (frey procedure) and duodenum-preserving resection of the pancreatic head (beger procedure). World J Surg. 2003:27(11):1217-30.

93. Keck T, Wellner UF, Riediger H, Adam U, Sick O, Hopt UT, et al. Long-term outcome after 92 duodenum-preserving pancreatic head resections for chronic pancreatitis: comparison of Beger and Frey procedures. J Gastrointest Surg. 2010;14(3):549-56.

94. Roch A, Teyssedou J, Mutter D, Marescaux J, Pessaux P. Chronic pancreatitis: A surgical disease? Role of the Frey procedure.World J Gastrointest Surg. 2014;6(7):129-35

95. Tan CL, Zhang H, Li KZ. Single center experience in selecting the laparoscopic Frey procedure for chronic pancreatitis. World J Gastroenterol. 2015;21(44):12644-52. 
96. Ho HS, Frey CF. The Frey procedure: local resection of pancreatic head combined with lateral pancreaticojejunostomy. Arch Surg. 2001;136(12):1353-8.

97. Gloor B, Friess H, Uhl W, Büchler MW. A modified technique of the Beger and Frey procedure in patients with chronic pancreatitis. Dig Surg. 2001;18(1):21-5.

98. Farkas G, Leindler L, Daróczi M, Farkas G Jr. Organ-preserving pancreatic head resection in chronic pancreatitis. Br J Surg. 2003:90(1):29-32.

99. Izbicki JR, Bloechle C, Broering DC, Kuechler T, Broelsch CE. Longitudinal $V$-shaped excision of the ventral pancreas for small duct disease in severe chronic pancreatitis: prospective evaluation of a new surgical procedure. Ann Surg. 1998;227(2):213-9.

100.Kutup A, Vashist Y, Kaifi JT, Yekebas EF, Izbicki JR. For which type of chronic pancreatitis is the «Hamburg procedure» indicated? J Hepatobiliary Pancreat Sci. 2010;17(6):758-62.

101. Yekebas EF, Bogoevski D, Honarpisheh H, Cataldegirmen G, Habermann $\mathrm{CR}$, Seewald $\mathrm{S}$, et al. Long-term follow-up in small duct chronic pancreatitis: A plea for extended drainage by «V-shaped excision» of the anterior aspect of the pancreas. Ann Surg. 2006;244(6):940-6; discussion 946-8.
102. Ray BS, Neill CL. Abdominal Visceral Sensation in Man. Ann Surg. $1947 ; 126(5): 709-23$

103. Hatori T, Imaizumi T, Harada N, Fukuda A, Suzuki M, Hanyu F, et al. Appraisal of the Imaizumi modification of the Beger procedure: the TWMU experience. J Hepatobiliary Pancreat Sci. 2010;17(6):752-7.

104. White TT, Slavotinek AH. Results of surgical treatment of chronic pancreatitis. Report of 142 cases. Ann Surg. 1979:189(2):217-24.

105. Warren WD, Millikan WJ Jr, Henderson JM, Hersh T. A denervated pancreatic flap for control of chronic pain in pancreatitis. Surg Gynecol Obstet. 1984;159(6):581-3.

106. Kaufman M, Singh G, Das S, Concha-Parra R, Erber J, Micames C, et al. Efficacy of endoscopic ultrasound-guided celiac plexus block and celiac plexus neurolysis for managing abdominal pain associated with chronic pancreatitis and pancreatic cancer. J Clin Gastroenterol. 2010:44(2):127-34.

107. Kesseli SJ, Smith KA, Gardner TB. Total pancreatectomy with Islet Autologus Transplantation: The Cure for Chronic Pancreatitis? Clin TransI Gastroenterol. 2015;6:e73.

108. Shah NS, Siriwardena AK. Variance in elective surgery for chronic pancreatitis. JOP. 2009;10(1):30-6. 